\title{
Vortical Flow Topology on Windward and Leeward side of Delta Wing at Supersonic Speed
}

\author{
Y. Younis ${ }^{1,2}$, A. Bibi ${ }^{1}$, A.U. Haque ${ }^{3}$ and S. Khushnood ${ }^{1}$ \\ ${ }^{1}$ Department of Mechanical Engineering, University of Engineering and Technology, Taxila. \\ ${ }^{2}$ Ali Ahmed Shah University College of Engineering and Technology, Mirpur, Azad Kashmir \\ ${ }^{3}$ Centers of Excellence in Sciences and Applied Technologies (CESAT), P O Box No. 2801, Islamabad \\ E-mail:yamin_596@yahoo.co.uk
}

(Received February 7, 2008; accepted November 29, 2008)

\begin{abstract}
CFD (Computational Fluid Dynamics) Analysis of Delta wing configurations used for supersonic flight regime is a key area of interest for flow control due to coherent vortical structures existing on the leeward side of wing. In the present research, a numerical study was carried out by using two different turbulence models at Mach No. 1.6, to identify the influence of turbulence modeling and wing on overall pressure distribution and onset of flow separation. Fully structured multi-block grid was used with the grid refinement near the wall to resolve the vortical flow structures accurately. Different turbulence models used were SA (Spalart-Allmaras) and k $\omega$-SST (Shear-stress Transport). An under prediction in pressure distribution was observed by using SA Turbulence model, in comparison with known experimental data at fixed Mach No. The analysis of results presented showed that the height of primary vortex increases by increasing the angle of attack and by keeping the Mach No. as fixed. Moreover, the vortex formation on cambered wing seems less pronounced as compared with that of sharp wing at high angle of attack.
\end{abstract}

Keywords: Flow Separation, Turbulence Modeling, Vortical Flow, Delta Wing, Structured Grid.

\section{INTRODUCTION}

The advantage of leading edge sweep and vortex formation in supersonic flight regime has lead to a detailed analysis over delta wings at high speeds. Sweep back of wing to a certain angle makes the normal angle of attack and normal Mach number relative to wing leading edge more important as compared to free stream parameters. This allows an ease in the limitation of maximum attainable speed, Bossuyt (2005). Another advantage of supersonic flight is the formation of leading edge vortices. These vortices increase the maximum lift coefficient by adding vortex lift. An extensive amount of experimental and computational study has been carried out on flow structures over delta wings by Ghaffari (2005). The 1940's marked the beginning of research work carried out on vortex formation at supersonic speeds. An abundant amount of research has been conducted since then in understanding these complex vortical flow structures as applied to civil and military aircrafts, missiles and space shuttles, Allen et al. (2003). Over the past three decades, delta wings have become a particular area of interest of researchers and scientists for investigation of vortical flow. The increasing amount of study includes effect of geometrical parameters such as sweep, leading edge radius, camber and wing thickness as well as flow parameters such as Mach number, Reynolds number and angle of attack; on flow configuration over the leeside of delta wings. The onset of flow separation, corresponding vortical flow, consequent vortex breakdown and their effect on wing aerodynamic characteristics,
Kato et al. (2005), stability, control, and maneuverability, Myose et al. (2004) have been the focus of research.

Along side experimentation, numerical methods have also become a popular mode of analysis (Rizzi et al. 1999, 2006, Rutten et al. 2004 and Munukka et al. 1999). The use of CFD not only aids in the further validation of experimentation but also assesses the capability of CFD codes to predict flow physics and vortical flow phenomena accurately. The present numerical analysis was carried out on two delta wings varying in geometry from sharp to cambered wing at Mach number 1.6 and at three different angles of attack. The computational data were compared with experimental results given in Bowen et al. (1996). Two turbulence models were also used to study their effect on prediction of pressure profile and onset of flow separation on delta wing.

\section{GEOMETRY AND GRID GENERATION}

Models of the two Delta wings were generated by using analytical expressions provided in Bowen et al. (1996). The wing profiles were created in the software GAMBIT ${ }^{\circledR}$ and are shown in Fig. 1. Comparison of the pressure coefficient between experimental and computational data was made at two $\mathrm{Z} / \mathrm{C}_{\mathrm{R}}$ locations as shown in Fig. 2. These are the locations on the upper and lower surface of wing where the pressure orifices are located. Where, $\mathrm{Z}$ is the distance from the apex in $\mathrm{Z}$-axis and $\mathrm{C}_{\mathrm{R}}$ is root chord distance. The two wings had a $65^{\circ}$ sweep, a root chord of 
18 inches and a semi-span of 8.3937 inches. The crosssectional shape can be varied to get sharp and chambered wings. The isometric views of the wing geometries are shown in Fig. 3.

Fully structured multi-block grids were used for all cases, with grid refinement near the wing surface to accurately capture the vortical flow physics. A sample surface grid view for the sharp wing is shown in Fig. 4. It can be seen that in case of sharp wing a dense mesh occurs in the apex region, whereas in camber wing the mesh is concentrated along the entire wing leading edge. The size of the grid domain was kept one $C_{R}$ upstream, one $C_{R}$ in radial and circumferential direction at upstream position and two $C_{R}$ in radial and circumferential direction at trailing edge. Total number of cells used for both wings were 1.35 million, which were obtained after excessive simulation for grid independent study. Analysis was carried out on only half wing models assuming symmetry conditions on root chord plane. The upper and lower surfaces of wing were specified as solid wall assuming no slip conditions. The remaining domain was specified as pressure-farfield for free stream inflow boundaries. An important factor to keep in mind during meshing is resolution of boundary layer. The boundary layer consists of three distinct layers. Starting from inner most layer these are linear sub-layer, buffer layer and log-law layer, Malalasekera et al. (1995). In order to capture flow within linear sub layer it is necessary to place the first node normal to wing surface such that value of $\mathrm{y}^{+}$ ranges from 1-5. Incase such low values of $\mathrm{y}^{+}$are not attainable and $\mathrm{y}^{+}>30$ then wall functions can be used for near wall treatment. For our analysis, due to limitations in computational resources, $\mathrm{y}^{+}$ranged from 20-30 and wall functions were employed to resolve near wall regions.

\section{Turbulence Modeling}

At very high Reynolds number the flow becomes turbulent and is characterized by unsteady and random variations in flow properties. The three dimensional Navier- Stokes equations used in Fluid Dynamics have the ability to resolve smallest scales of motion. Use of these complete equations in CFD is called Direct Numerical Simulation (DNS), which requires such extensive computational resources that their applicability is not feasible at present. Instead Reynolds Average Navier Stokes (RANS) equations are used. The RANS equations are similar to Navier-Stokes equations except that they are time averaged with fully modeled turbulent scales. A key advantage of RANS approach is a reduction in computational resources, which has made it a common choice of adoption in practical industrial applications. Research however has revealed that RANS based turbulence models under predict the onset of flow separation at high angles of attack, Hajek et al. (2002).

The Spallart-Allmaras (S-A), k- $\varepsilon$ and k- $\omega$ models all based on RANS use the Boussinesq hypothesis for computing turbulent viscosity. The disadvantage of this hypothesis is that it assumes turbulent viscosity to be a scalar quantity. In flow phenomena where anisotropy of turbulence is dominated, Reynolds Stress Model (RSM) is superior, but requires more computation resources. However, the Boussinesq hypothesis based models have been found to perform well in many situations and computational expenses of RSM are not yet justified.

One of the key areas of CFD research is to find the most feasible turbulence model, Leschziner (2006). However, to present day, no singular turbulence model has been found to be satisfactory under all applications. The one-equation and two-equation RANS based turbulence models with certain modifications have been used to effectively simulate flow behavior over delta wings (Oskam et al. 2002, Gordnier 1996 and Chaderjian et al. 1998). The oneequation Spallart-Allmaras model and two-equation modified k- $\omega$ SST (Shear Stress Transport) model were used in the present numerical analysis. The S-A model is easy to implement and requires lesser computational resources. In addition it also has the capability of employing wall functions for near wall treatment. The model however is not suitable in cases where accurate simulation of turbulent flow is required or when near wall gradients are high. It is one equation model, which solves a single partial differential equation for variable $\tilde{v}$, which is related to the turbulent viscosity. This model can directly be applied through out the boundary layer if the near wall mesh is fine enough to resolve the gradients. In SA model turbulent eddy viscosity is not specified with characteristic velocity and length scales, rather then solves by a transport equation, which is given below:

$$
\begin{aligned}
& \frac{\partial \tilde{v}}{\partial t}+\frac{\partial \bar{u}_{j} \tilde{v}}{\partial x_{j}}=C b 1 \\
& {\left[1-f_{t 2}\right] \tilde{S} \tilde{v}+\frac{1}{\operatorname{Re}} \frac{1}{\sigma} \frac{\partial}{\partial x_{j}}\left[(v+\tilde{v}) \frac{\partial \tilde{v}}{\partial x_{j}}\right]} \\
& -\frac{1}{\operatorname{Re}} C w 1 f w\left[\frac{\tilde{v}}{d}\right]^{2}+\frac{1}{\operatorname{Re}} \frac{C b 2}{\sigma} \frac{\partial \tilde{v}}{\partial x_{j}}
\end{aligned}
$$

Where $\tilde{S}$ is strain vorticity vector, $C b 1$ and $C b 2$ are constants and $C w 1$ and $f w$ are auxiliary functions. Gordnier (1996) combined the k- $\varepsilon$ and $k-\omega$ models on a way that would allow them to be used in the regions where they attain the best advantage. This model is quite similar to the k- $\omega$ BSL model, except that the constants for the inner model are slightly changed and the definition of eddy viscosity was redefined as follows:

$$
\mu_{T}=\operatorname{Re} \cdot \min \left[\frac{\rho k}{\omega} \frac{a_{1} \rho k}{\Omega F_{2}}\right]
$$

The k- $\omega$ SST turbulence model is a two-equation model solving for turbulent kinetic energy $\mathrm{k}$ and specific turbulent dissipation rate $\omega$. It is a modification of standard k- $\omega$ model and used for more accurate simulation of a wider class of flow problems. On the other hand, the model is more expensive to implement (due to two equations) as compared to S-A model.

\section{Mathematical Modeling}

The commercially available software, FLUENT $\left({ }^{\circledR}\right.$ was used to carryout the numerical simulations. This finite volume based solver uses RANS equations coupled with turbulence models to simulate the flow for different aerospace applications. A coupled explicit solver was used to run all the solutions. Although an implicit solver requires lesser number of iterations, but the time required per iteration is also more. Due to the difficulties in getting the converged solutions, the coupled explicit solver was selected and the convergence difficulties were handled by 
changing the CFL number. Initially the CFL was kept low 0.1 and was gradually increased with iterations upto a final value of 1 . In order to achieve a better and more accurate solution and to resolve the shockwave features, the Second Order Upwind Scheme was used, and the convergence criteria was also reduced once convergence was achieved with default values. Density was calculated by ideal gas law and viscosity by Sutherland's law.

In the numerical procedure, the flow parameters were kept constant at Mach number 1.6, Reynolds number $2 \times 10^{6}$ per foot, stagnation temperature $125^{\circ} \mathrm{F}$, and stagnation pressure 1079 psf. Each wing was tested at three nominal angles of attack $0^{\circ}, 5^{\circ}$ and $9^{\circ}$. The exact angle of attack used for each wing was kept similar to that provided by McMillin (1996). Hence for sharp wing the angles of attack were $0.25^{\circ}, 5.25^{\circ}$ and $9.28^{\circ}$ and for chamber wing $0.21^{\circ}, 5.22^{\circ}$ and $9.23^{\circ}$ respectively.

\section{Results AND Discussion}

A detailed computational analysis was carried out on the two delta wings geometries to study the effect of turbulence model as well as the influence of wing profile on pressure distribution and vortex formation on leeside of delta wing. Simulations were carried out for both sharp and cambered wings at nominal angle of attack of $0^{\circ}$, with SA Turbulence model.

The resultant pressure coefficients data as compared with experimental data at two $\mathrm{Z} / \mathrm{C}_{\mathrm{R}}$ locations is shown in Fig. 5 and 6 for sharp and cambered wings respectively. It can be seen that there is a good agreement between experimental and computational data in both cases. Also evident from the pressure coefficients plots is that at low angles of attack there is no flow separation. Hence the SA Turbulence model is found to be suitable in cases where flow remains attached. Also the points on the graph are clustered, showing that there in no noticeable difference of pressure between upper and lower surfaces at negligible angle of attack. The next series of runs were carried out for both sharp and cambered wings at nominal angle of attack of $5^{\circ}$ with SA turbulence model. The resultant pressure coefficient plots were compared well with experimental data at $\mathrm{Z} / \mathrm{C}_{\mathrm{R}}=0.33$ for both sharp wing and cambered wing and is shown in Fig.7.

The results show that SA Turbulence model was unable to predict the flow and capture vortex formation occurring at high angles of attack. This deficiency of SA model for vortical flow simulations over delta wing is associated with the choice of length scale for separated flows. Whereas, the two equation turbulence models, like SST model does not require the explicit specification of length scales. The SA Turbulence model was therefore found unsuitable at higher angles of attack and it was decided to carry out further analysis with $\mathrm{k} \omega$-SST Turbulence model. The pressure coefficient plots as compared with experimental data by using $\mathrm{k} \omega$-SST Turbulence model at nominal angle of attack of $5^{\circ}$ for both sharp and cambered wing at $Z / C_{R}=0.33$ is shown in Fig. 8. In this case a good agreement in results was found by use of $\mathrm{k} \omega$-SST Turbulence model. The contours of pressure coefficient and surface streamline visualizations for both Turbulence models is shown in Figs. 9 and 10 for sharp and cambered wings respectively. It is clear from the sectional plots of co-efficient of pressure, shown in Fig. 9 that height of the primary vortex was found to be increasing as we go downstream from the apex.
Moreover, the comparison of turbulence modeling in this figure reveal that the SST model has captured the separation and reattachment line as compared wit the flow physics captured by using SA model. Therefore, these visualization results clearly indicate the ability of k $\omega$-SST Turbulence model to capture vortical flow at higher angles of attack as compared to SA Turbulence model. Surface flow visualization on two different wings (Fig. $9 \&$ Fig. 10) shows that the primary reattachment line moves inward in case of chambered wing in comparison with sharp wing at same angle of attack. No separation was observed on the windward side of wings and a good quantitative comparison with the experimental measurements was obtained in all cases at different angles of attack.

The last series of simulations were carried out at nominal angle of attack $9^{\circ}$ for both sharp and cambered wings using $\mathrm{k} \omega$-SST Turbulence model. The pressure coefficient plots as compared with experimental data at two $\mathrm{Z} / \mathrm{C}_{\mathrm{R}}$ locations are shown in Fig. 11. The plots show good agreement between experimental and computational data. However, the computational results of sharp delta wings have noticeable under prediction in the onset of flow separation and the quantity of flow separation at two different $\mathrm{Z} / \mathrm{C}_{\mathrm{R}}$ locations. These results may be improved by further clustering the grid cells close to the wall for better resolution of the boundary layer features by keeping $\mathrm{y}^{+}$of the order of one or by using the non-equilibrium wall function to handle the adverse pressure gradient close to the wall at high angle of attack. The total pressure contours at four $\mathrm{Z} / \mathrm{C}_{\mathrm{R}}$ locations for both sharp and cambered wings at nominal angle of attack $9^{\circ}$ is shown in Fig. 12. The visualizations clearly indicate an increase in strength of vortices with increase in distance on leeside of delta wing. It is also evident that the strength of vortices incase of sharp wing is greater as compared to cambered wing. Hence leading edge radius and camber cause a reduction in strength of vortices. Fig. 13 and 14 show effect of increase in angle of attack for both sharp and cambered wing respectively. From the contours of pressure coefficient it is evident that strength of vortices increases by increasing angle of attack. Similar effect was captured on different delta wings geometries at subsonic speed (Chaudhry et al. 2006 and 2007). Moreover, the separation and reattachments lines in both cases reveal the absence of secondary and tertiary vortices. Future work will include the numerical analysis of vortical flow on elliptic wing under the same flow conditions to identify the role of wing geometry for active flow control.

\section{CONCLUSION}

From the extensive amount of numerical experiments conducted by using the $\mathrm{k} \omega$-SST Turbulence model and the quantitative comparison with experiments, it was concluded that this turbulence model is more suitable to capture vortical flow at higher angles of attack as compared to SA Turbulence model. A study of effect of flow parameters indicated that an increase in angle of attack caused an increase in strength of vortex formation on leeside of delta wing. This strength of vortices, however, was less pronounced incase of cambered wing as compared to sharp wing. Thus the effect of leading edge radius and camber is to reduce the strength of vortices. 


\section{ACKNOWLEDGMENT}

The authors are indebted to the facilities provided by CAD Lab 1, UET, Taxila. Without them such extensive computational analysis would not be possible. Sincere thanks are also extended to Aero Consulting Pakistan for providing the computational support for flow visualization.

\section{REFERENCES}

Bossuyt, B.V. (2005). Experimental Investigation of the Vortex Flow over the Apex of a Delta Wing. Dissertation of Delft University of Technology, the Netherlands, February 8.

Crippa, S. and A. Rizzi (2006). Numerical Investigation of Reynolds Number Effects on a Blunt Leading-Edge Delta Wing. 24th AIAA Applied Aerodynamics Conference, AIAA-2006-3001, June 2006.

Dol, H.S., J.C. Kokand, and B. Oskam (2002). Turbulence Modeling for Leading-Edge Flows. $40^{\text {th }}$ Aerospace Sciences Meeting \& Exhibit, AIAA 2002-0843, January.

Ghaffari, F. (2005). Turbulent Vortex-Flow Simulation over a $65^{\circ}$ Sharp and Blunt Leading-Edge Delta wing at Subsonic Speeds. NASA/TM-2005-213781.

Gordnier, R.E. (1996). Computational Study of a Turbulent Delta-Wing Flow field using Two-Equation Turbulence Models. 27 $7^{\text {th }}$ AIAA Fluid Dynamics Conference, AIAA, 96-2076, June.

Gortz, S., A. Rizzi and K. Munukka (1999). Computational Study of Vortex Breakdown over Swept Delta Wings. AIAA-99-3118.

Haque, A.U. and J. Khawar, A. Jabbar and S.R. Chaudhry (2007). Numerical Investigation of Leading Edge vortical Flow on 65 degree Delta Wing at Subsonic Speed. $5^{\text {th }}$ International Bhurban Conference on Applied Sciences and Technology, January 8-11, 2007, Islamabad, Pakistan.

Haque, A.U., J. Khawar, A. Jabbar and S.R. Chaudhry (2006). Effect of Turbulence Modeling on Leading edge Vortical Flow. International Conference on Computational Methods in Sciences and Engineering (ICCMSE 2006), 27 Oct-1 Nov 2006. Copy of paper already published in a special volume of the Lecture Series on Computer and Computational Sciences (VSP/Brill).

Heron, I. and R.Y. Myose (2004). On the Impingement of a Von Karman Vortex Street on a Delta Wing. $22^{\text {nd }}$ Applied Aerodynamics Conference and Exhibit, AIAA 2004-4731.

Kawazoe, H. and S. Kato (2005). Effects of Leading Edge Separation Vortex of Flexible Structure Delta Wing on its Aerodynamic Characteristics. Int. Conf. on Jets, Wakes and Separated Flows, ICJWSF-2005.
Leschziner, M.A. (2006). Modeling Turbulent Separated Flow in the Context of Aerodynamic Applications. Fluid Dynamics Research 38(2006), 174-210.

McMillin, S.N., J.E. Byrd, D.S. Parmer, G.M.B. O'Connor, D.K. Forrest, and S. Bowen (1996). Surface-Pressure and flow-Visualization Data at Mach number of 1.6 for three $65^{\circ}$ Delta Wings Varying in Leading-Edge Radius and Camber. NASA Technical Memorandum 4673, December 1996.

Moigne, Y.L. and A. Rizzi (1999). Time Accurate Simulations of High- Alpha Aerodynamics for Pitching Delta Wings. RTO-MP-069(I).

Morton, S.A., J.R. Forsythe, A.M. Mitchell, and D. Hajek (2002). DES and RANS Simulation of Delta Wing Vortical Flows. $40^{\text {th }}$ Aerospace Sciences Meeting \& Exhibit, A02-13906, January.

Murman, S.M. and N.M. Chaderjian (1998). Application of Turbulence Models to Separated High-Angle-ofAttack Flows. AIAA 98-37280.

Tricoche, X., C. Garth, T. Bobach, G. Scheuermann and M. Rutten (2004). Accurate and Efficient Visualization of Flow Structures in a Delta Wing Simulation. $34^{\text {th }}$ AIAA Fluid Dynamics Conference and Exhibit, AIAA 2004-2153, July 2004.

Versteeg, H.K. and W. Malalasekera (1995). An Introduction to Computational Fluid Dynamics- The Finite Volume Method. Essex, England: Longman Scientific \& Technical.

Wood, R.M., F.J. Wilcox Jr., S.X.S. Bauer, and J.M. Allen (2003). Vortex Flows at Supersonic Speeds. NASA/TP-2003-211950. 


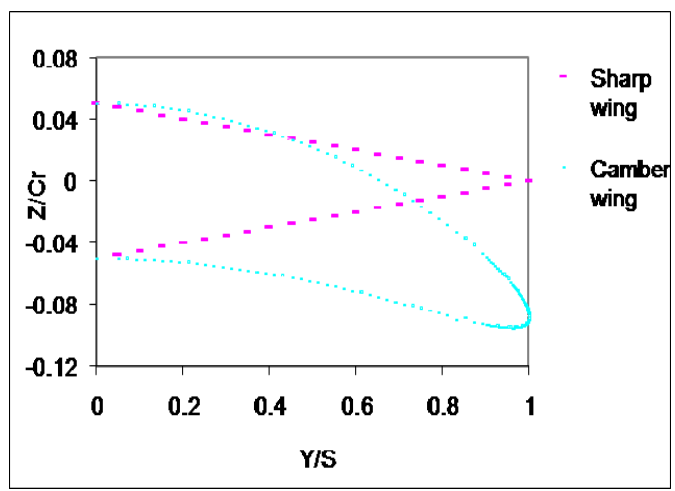

Fig. 1. Wing leading edge profiles

a) Sectional view of sharp wing

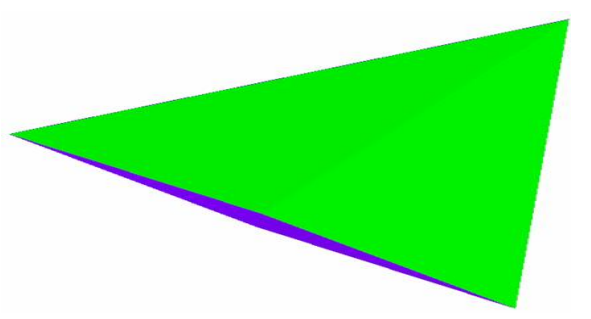

c) Sharp wing

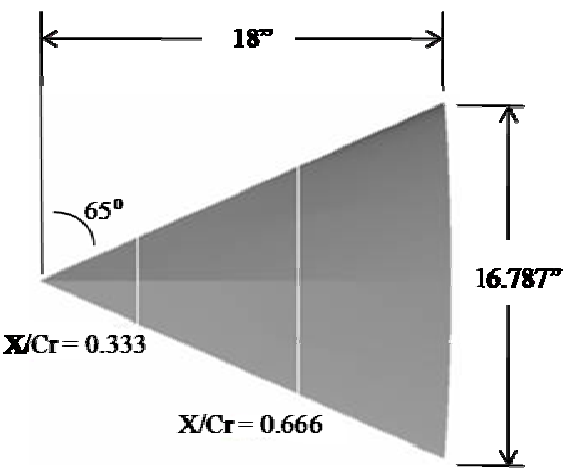

Fig. 2. Position of Pressure Measurement Station on wing with dimensions

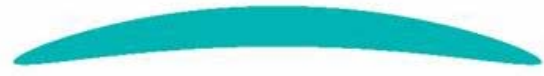

b) Sectional view of cambered wing

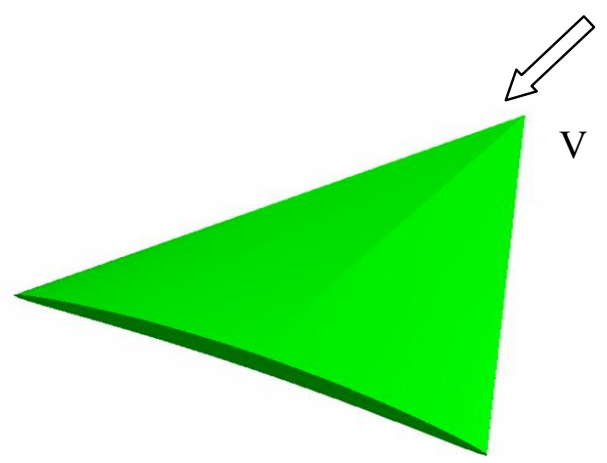

d) Cambered wing

Fig. 3. Isometric views of sharp and chambered wings

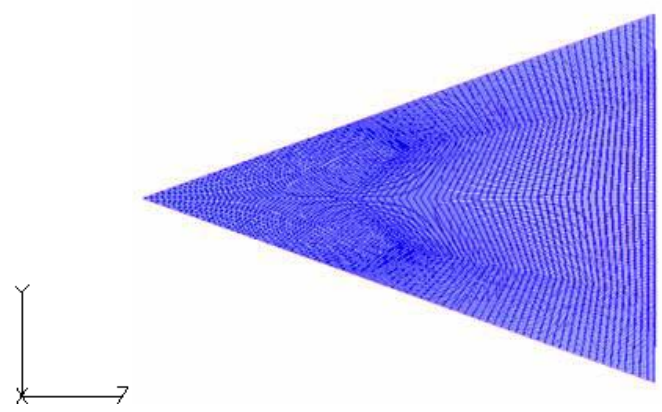

Fig. 4. Surface Grid View of Sharp Wing 


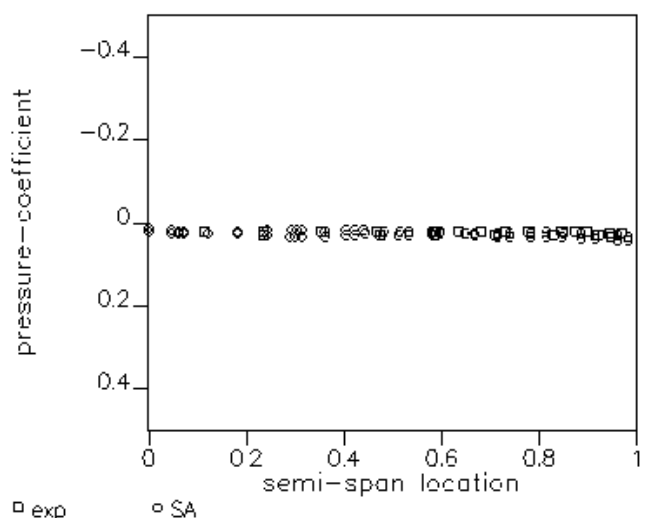

a) at $\mathrm{Z} / \mathrm{C}_{\mathrm{R}}=0.33$

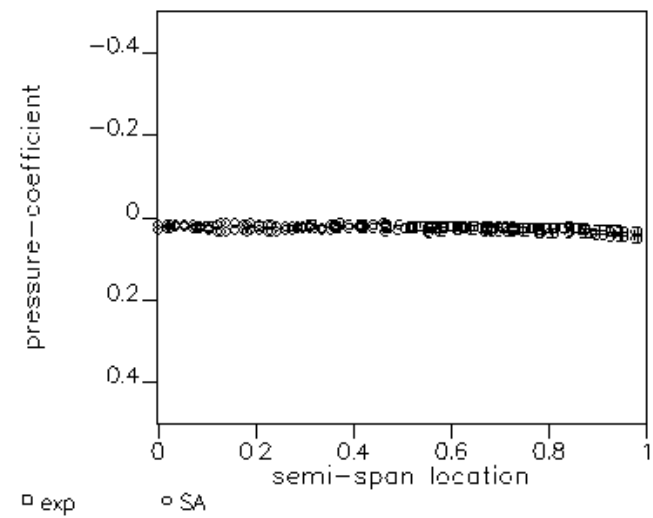

b) at $\mathrm{Z} / \mathrm{C}_{\mathrm{R}}=0.66$

Fig. 5. Results of Co-efficient of pressure of sharp wing with SA Turbulence model and its co-relation with experimental data at $\mathrm{M}=1.6 \alpha=0.25^{\circ}, \mathrm{Re}=2 \times 10^{6}$ at different $\mathrm{Z} / \mathrm{C}_{\mathrm{R}}$ Locations

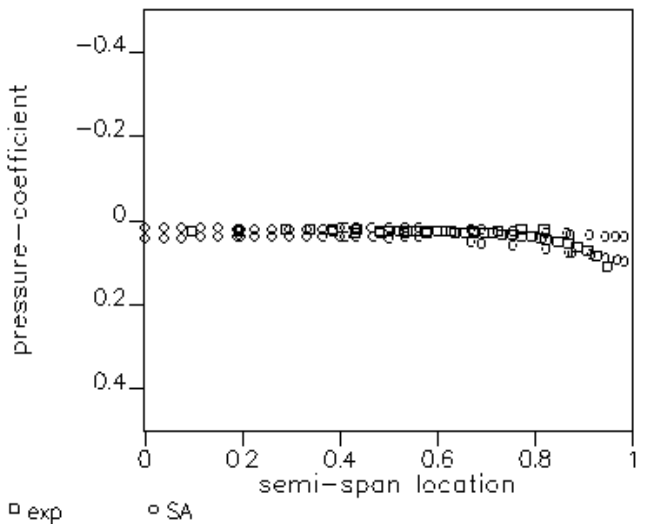

a) at $\mathrm{Z} / \mathrm{C}_{\mathrm{R}}=0.33$

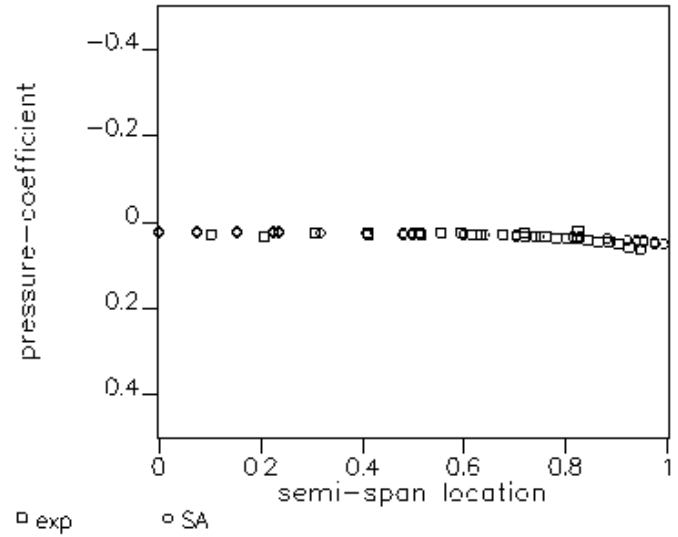

b) at $\mathrm{Z} / \mathrm{C}_{\mathrm{R}}=0.66$

Fig. 6. Results of Co-efficient of pressure of cambered wing with SA Turbulence model and its co-relation with experimental data at $\mathrm{M}=1.6 \alpha=0.21^{\circ}, \mathrm{Re}=2 \times 10^{6}$ at different $\mathrm{Z} / \mathrm{C}_{\mathrm{R}}$ locations

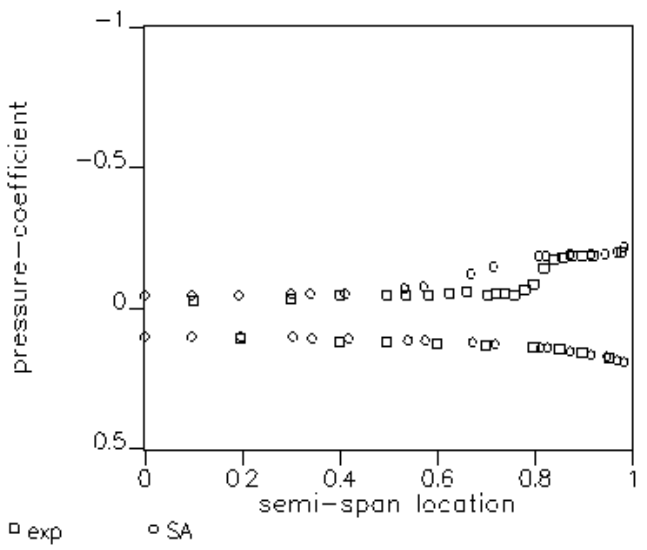

a) Sharp wing

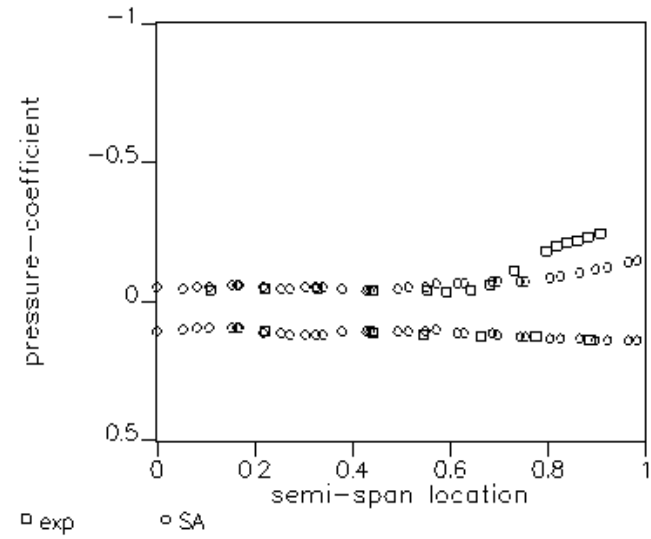

b) Camber wing

Fig. 7. Comparison of Co-efficient of pressure of two wing profiles with SA Turbulence model and its co-relation with experimental data at $\mathrm{M}=1.6 \alpha=5.25^{\circ}$ (sharp wing) and $\alpha=5.17^{\circ}$ (camber wing), $\mathrm{Re}=2 \times 10^{6}$ at $\mathrm{Z} / \mathrm{C}_{\mathrm{R}}=0.33$ 


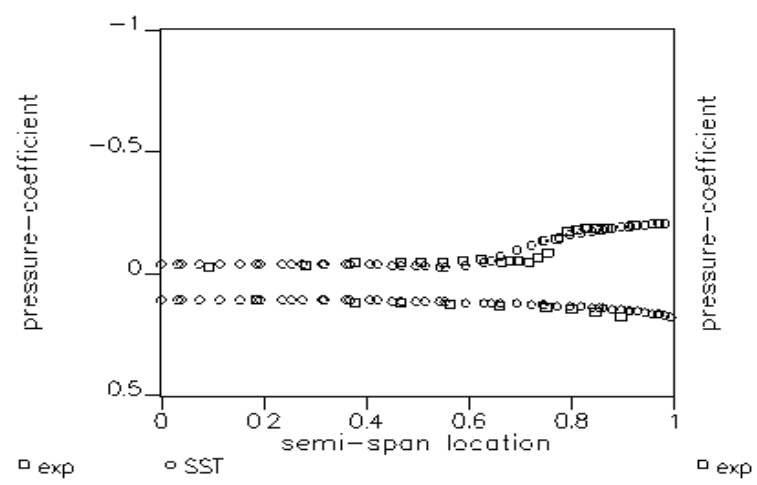

a) Sharp wing

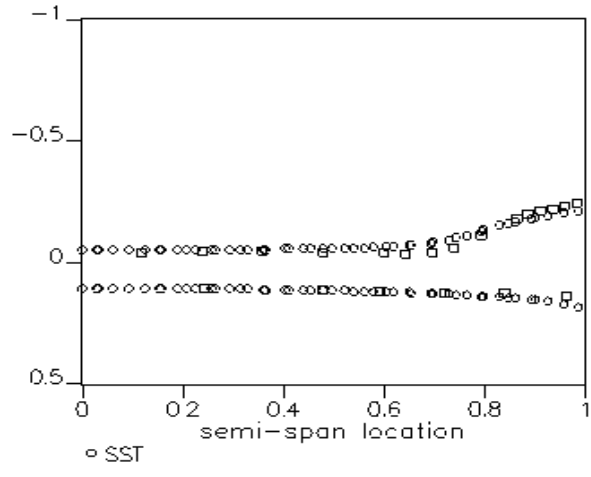

b) Camber wing

Fig. 8. Comparison of Co-efficient of pressure of two wing profiles with SST Turbulence model and co-relation with experimental data at $M=1.6 \alpha=5.25^{\circ}$ (sharp wing) and $\alpha=5.17^{\circ}$ (camber wing), $\mathrm{Re}=2 \times 10^{6}$ at $\mathrm{Z} / \mathrm{C}_{\mathrm{R}}=0.33$

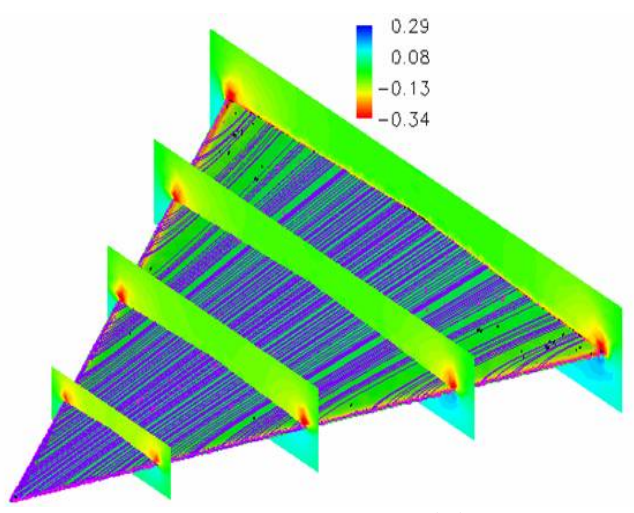

a) SA Model

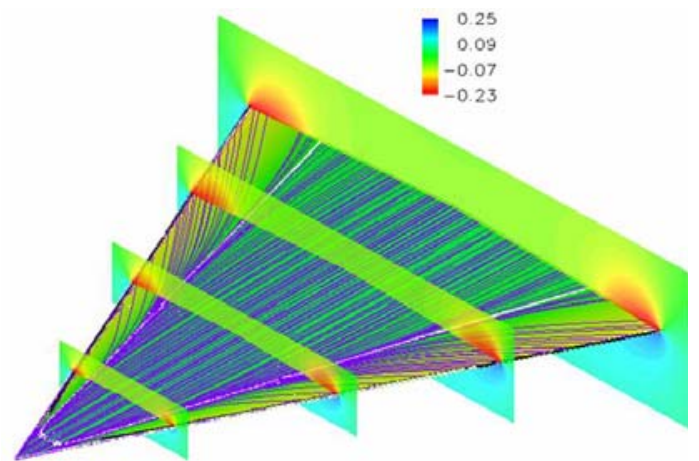

b) SST Model

Fig. 9. Contour plots of co-efficient of pressure for sharp wing for two different turbulence models $\alpha=5.25^{\circ}$ at four different $\mathrm{Z} / \mathrm{C}_{\mathrm{R}}$ locations

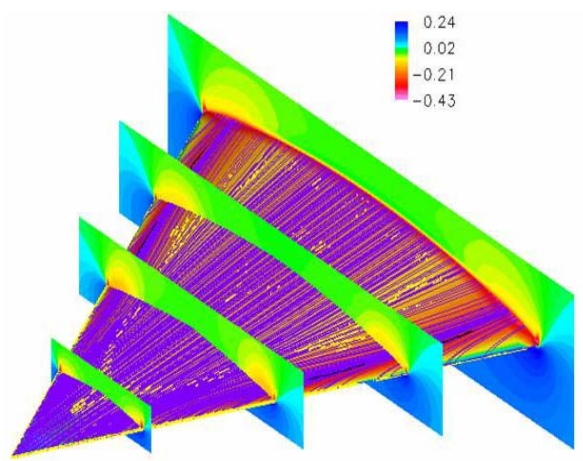

a) SA Model

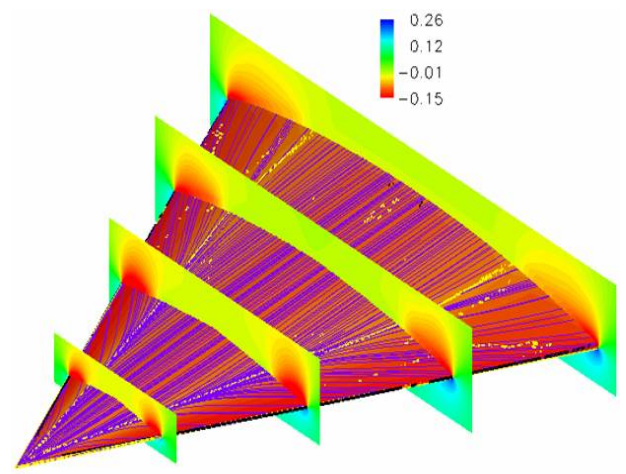

b) SST Model

Fig. 10. Contour plots of co-efficient of pressure for two different turbulence models for cambered wing for $\alpha=5.17^{\circ}$ at four different $\mathrm{Z} / \mathrm{C}_{\mathrm{R}}$ locations 


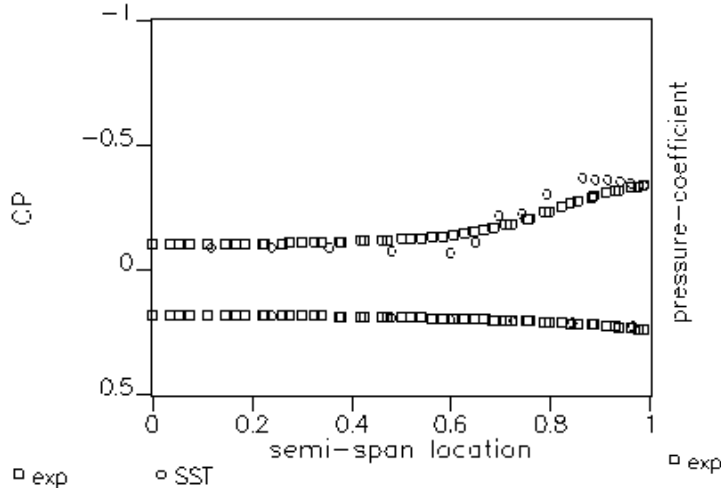

a) Sharp wing $\left(\mathrm{Z} / \mathrm{C}_{\mathrm{R}}=0.33\right)$

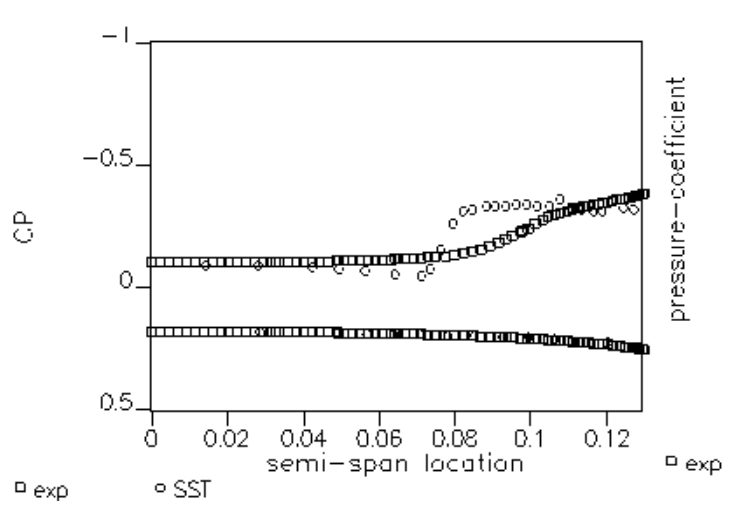

c) Sharp wing $\left(\mathrm{Z} / \mathrm{C}_{\mathrm{R}}=0.66\right)$

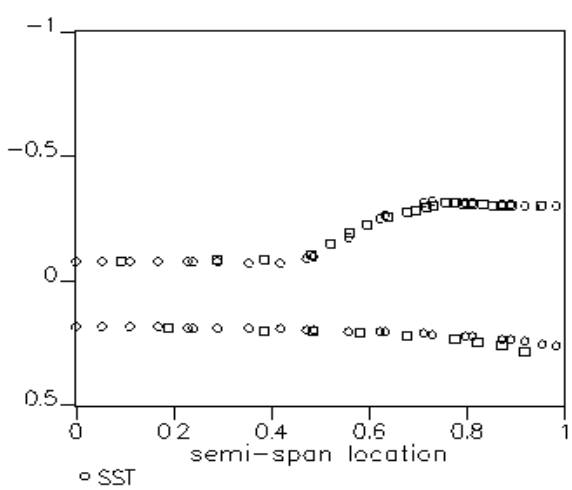

b) Camber wing $\left(\mathrm{Z} / \mathrm{C}_{\mathrm{R}}=0.33\right)$

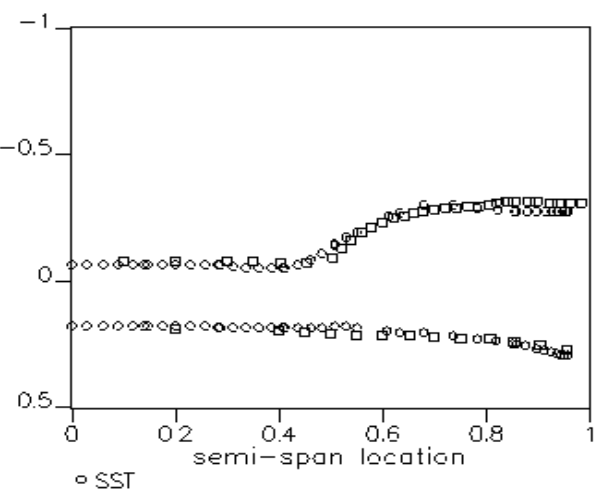

d) Camber wing $\left(\mathrm{Z} / \mathrm{C}_{\mathrm{R}}=0.66\right)$

Fig. 11. Comparison of Co-efficient of pressure of two wing profiles with SST Turbulence model and co-relation with experimental data at $\mathrm{M}=1.6 \alpha=9.28^{\circ}$ (sharp wing) and $\alpha=9.23^{\circ}$ (camber wing), $\mathrm{Re}=2 \times 10^{6}$ at different $\mathrm{Z} / \mathrm{C}_{\mathrm{R}}$ locations

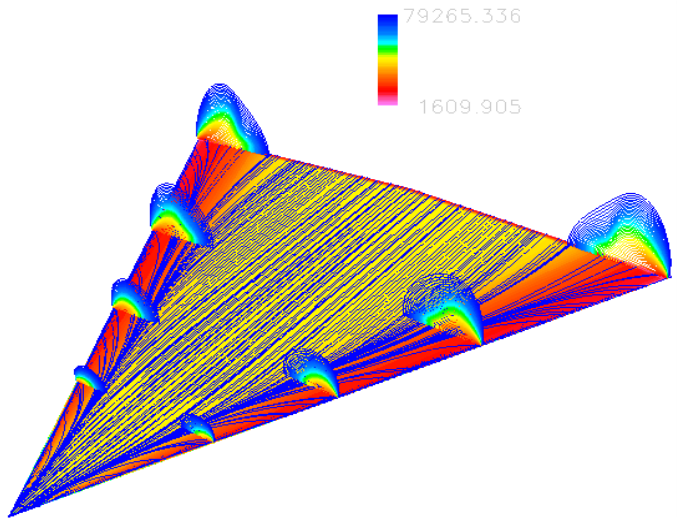

a) Sharp wing

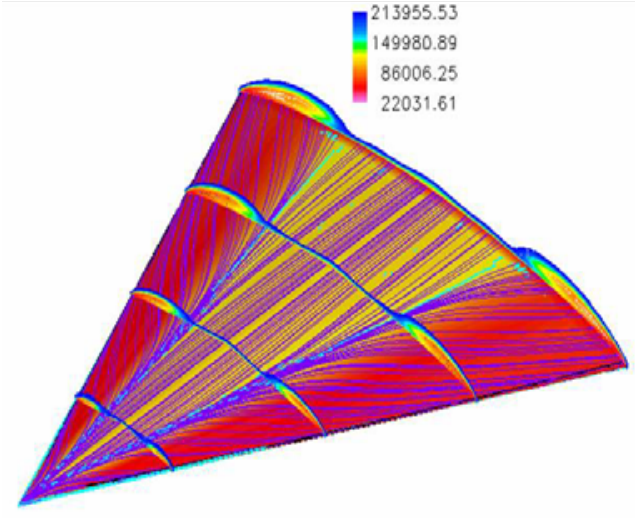

b) Cambered wing

Fig. 12. Total pressure contours at $\alpha=9.28^{\circ}$ (sharp wing) and $\alpha=9.23^{\circ}$ (camber wing) by using k- $\omega$ SST turbulence model at different $\mathrm{Z} / \mathrm{C}_{\mathrm{R}}$ locations for two wings along with surface streamlines 


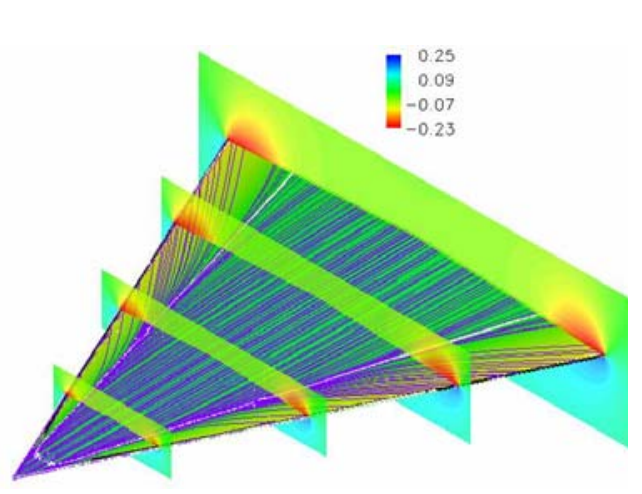

a) $\alpha=5.25^{\circ}$

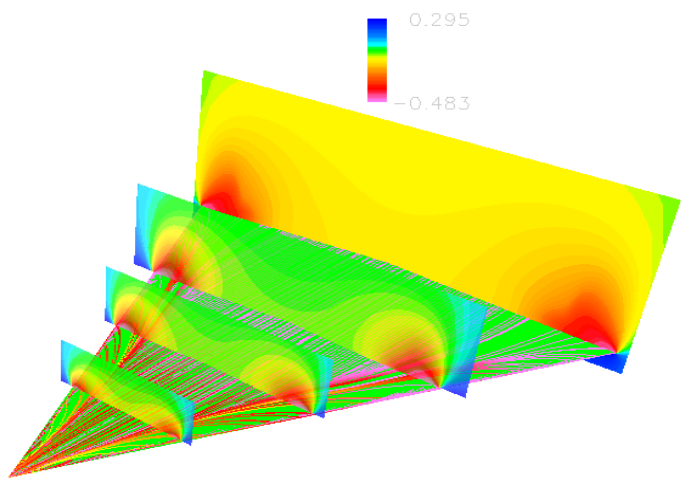

b) $\alpha=9.28^{\circ}$

Fig. 13. Computed surface streamlines and coefficient of pressure of sharp wing using k- $\omega$ SST turbulence model for $\alpha=5.25^{\circ}$ and $\alpha=9.28^{\circ}$, at $\mathrm{M}=1.6$ and $\mathrm{Re}=2 \times 10^{6}$

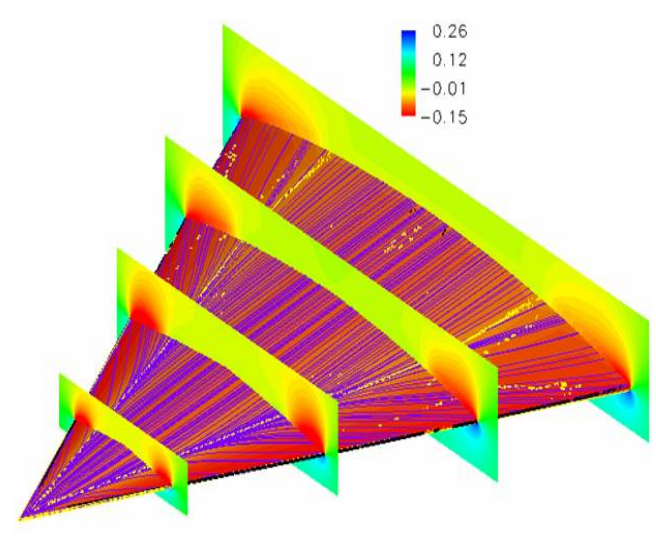

a) $\alpha=5.17^{\circ}$

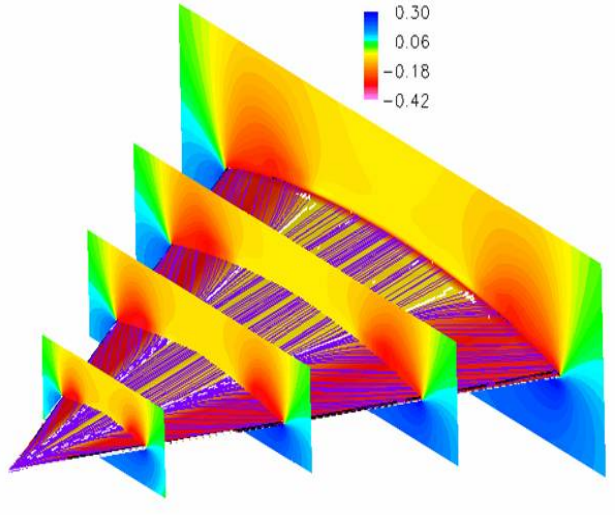

b) $\alpha=9.23^{\circ}$

Fig. 14. Computed surface streamlines and coefficient of pressure of camber wing using k- $\omega$ SST turbulence model for $\alpha=5.17^{\circ}$ and $\alpha=9.23^{\circ}$, at $\mathrm{M}=1.6$ and $\mathrm{Re}=2 \times 10^{6}$ 